\title{
Six and seven loop Konishi from Lüscher corrections
}

\author{
Zoltán Bajnok $^{a}$ and Romuald A. Janik ${ }^{b}$ \\ ${ }^{a}$ MTA Lendület Holographic QFT Group, Wigner Research Centre, \\ H-1525 Budapest 114, P.O.B. 49, Hungary \\ ${ }^{b}$ Institute of Physics, Jagiellonian University, \\ ul. Reymonta 4, 30-059 Kraków, Poland \\ E-mail: bajnok.zoltan@wigner.mta.hu, romuald@th.if.uj.edu.pl
}

ABSTRACT: In the present paper we derive six and seven loop formulas for the anomalous dimension of the Konishi operator in $\mathrm{N}=4$ SYM from string theory using the technique of Lüscher corrections. We derive analytically the integrand using the worldsheet S-matrix and evaluate the resulting integral and infinite sum using a combination of high precision numerical integration and asymptotic expansion. We use this high precision numerical result to fit the integer coefficients of zeta values in the final analytical answer. The presented six and seven loop results can be used as a cross-check with FiNLIE on the string theory side, or with direct gauge theory computations. The seven loop level is the theoretical limit of this Lüscher approach as at eight loops double-wrapping corrections will appear.

KeYwords: AdS-CFT Correspondence, Integrable Field Theories, Supersymmetric gauge theory

ARXIV EPRINT: 1209.0791 


\section{Contents}

1 Introduction 1

2 Asymptotic Bethe Ansatz up to 7-loops 4

3 Lüscher type corrections $\quad \mathbf{5}$

3.1 Direct energy correction 5

$\begin{array}{lll}3.2 & \text { Correction to the asymptotical BA } & 9\end{array}$

4 Cross-checks for the integrand $\quad 11$

5 The evaluation of the multiparticle Lüscher correction 12

$\begin{array}{lll}6 & \text { The final result and conclusions } & 14\end{array}$

\section{Introduction}

The Konishi operator is the simplest nonprotected local operator in $\mathcal{N}=4$ Super-YangMills theory. For this reason, its properties serve as a theoretical laboratory in our quest for the complete solution of $\mathcal{N}=4 \mathrm{SYM}$ at any coupling. The relative simplicity of its structure and properties allows us to probe them using various methods - including direct gauge theoretical computations and computations on the string side of the AdS/CFT correspondence. On both sides of the AdS/CFT duality there are various ways (sometimes conceptually quite different) of performing these computations and the agreement of the computations was always a strong confirmation of the respective methods. Apart from the above mentioned comparisons between different computations for the Konishi operator, there are also sometimes some quite nontrivial internal consistency checks within one particular method (e.g. the cancellation of dynamical pole contributions in the Lüscher correction method, the general transcendentality structure of the final result) which serve as a very strong and nontrivial constraint on the ingredients entering the computation like the fine structure of the S-matrix and its most complicated component - the so-called 'dressing phase' etc.

The anomalous dimension of the Konishi operator up to three loops is beautifully described by the Asymptotic Bethe Ansatz (ABA) of Beisert and Staudacher within the spin-chain approach [1].

The four-loop anomalous dimension of the Konishi operator was particularly important as it was only starting from this order of perturbation theory that deviations from the ABA appear due to a new topological class of Feynman diagrams - so-called 'wrapping interactions'. These effects cannot be understood within the spin-chain point of view and their description neccessitates the use of the string $\sigma$-model in $A d S_{5} \times S^{5}$. The "wrapping 
corrections' are then naturally identified [2] with finite-size Lüscher virtual corrections charactersitic of a two-dimensional worldsheet QFT. The agreement of direct gauge theory perturbative computations [3, 4] and the string $\sigma$-model worldsheet QFT computation [5] showed effectively that the gauge theory spin chain breaks down exactly in the way expected from a two-dimensional QFT on a cylinder i.e. string theory.

The five-loop order was computed initially only from the string Lüscher corrections [6]. The main motivation for this computation was that at five loops many more nontrival ingredients of the string $\sigma$-model computation start to appear, in particular an infinite set of coefficients of the dressing phase and virtual modifications of the quantization conditions for the constituents of the Konishi state. Here the test of these ingredients were initially just the internal cross-checks of the Lüscher computation like the cancellation of so-called dynamical pole contributions and a simple transcendental structure of the final result. A second motivation for this computation was to provide a cross-check for the exact sets of Thermodynamic Bethe Ansatz equations proposed for the spectrum of $\mathcal{N}=4 \mathrm{SYM}$ theory [8-10]. Subsequently full agreement was found, first numerically [11] and then analytically [12].

It is worth mentioning that the Konishi operator is the first member of an important family of so-called twist-two operators, whose anomalous dimensions obey the still mysterious maximal transcendentality principle [13] and intricate relations with BFKL. In [14] it was found that the Asymptotic Bethe Ansatz answer is inconsistent with BFKL starting from four loop level, with the discrepancy being attributed to the then unknown wrapping corrections. The wrapping corrections for these operators have been computed on the string side using Lüscher corrections at four- and five-loop level $[15,16]$, restoring consistency with BFKL expectations.

Lüscher corrections for multiparticle states include only the single wrapping contribution. However the sets of TBA equations [8-10] proposed later, provide a method of obtaining the exact anomalous dimension for any coupling thus resumming all multiple wrapping effects at once. Numerical computations of the Konishi anomalous dimension for a wide range of couplings have been performed in [17] and confirmed in [18]. Subsequently large coupling asymptotic fits to the numerical data have been shown to agree with string theory computations [19-21].

The reason for returning to the six- and seven-loop Konishi anomalous dimension using the Lüscher correction approach in the present paper has been twofold. Firstly, on the gauge theory side new techniques have been developed which allowed for a direct gauge theory computation at the five-loop level [22] and the verification of the previous string computation [6]. The authors informed us that a six-loop gauge theory computation using their methods was within reach and expressed interest in having a string theory cross-check. However, numerical methods of solving TBA equations are not precise enough to extract such high loop level coefficients, thus making the application of the conventional Lüscher computation an attractive alternative when applicable.

Secondly, the infinite set of TBA equations has been recast in a form of a finite set of nonlinear integral equations (FiNLIE) [23, 24], which makes a weak coupling expansion practically possible - however it is still very complex. This endeavor is now almost 
finalized [25], and the present Lüscher computation may provide a nontrivial cross-check.

For the above reasons we have embarked on computing the six and seven loop anomalous dimension of the Konishi operator using the multiparticle Lüscher correction formulas of $[5,6]$. The seven loop level is the theoretical limit of this approach as at eight loops double-wrapping will appear which is not taken into account by these formulas.

The general structure of the result of the multiparticle Lüscher correction formula is

$$
\Delta E_{6,7-\text { loop }}=-\sum_{Q=1}^{\infty} \frac{1}{2 \pi} \int_{-\infty}^{\infty} d q f_{6,7-\text { loop }}(q, Q)
$$

where the integrand can be obtained in a relatively straightforward way. At $n$-loop the integrand contains the $(n-4)^{t h}$ power of the polygamma function originating from the dressing phase. Evaluating the integral by residues $[6,7]$ derivatives of the polygamma function at integers values appear which, after summation for $Q$, can be expressed in terms of multiple zeta values (MZVs). At $n$-loop MZVs of depth $n-3$ appear [7]. Interestingly, irreducible MZVs showing up at various stages of the calculations cancel completely leaving only products of simple zeta functions. We do not have an understanding of this phenomenon yet, but will assume that similar structure will appear for the Konishi operator, too. Of course, the fact that our final results can be fitted to a very high accuracy without using irreducible MZV's strongly supports this assumption.

In the present case we have decided to bypass the technical steps above, as at six and seven loops they would be prohibitively complicated. We derive just the analytical expression for the integrand $f(q, Q)$ and perform the resulting integral and sum seminumerically to a very high precision ${ }^{1}$ which allows us to determine the coefficients of the zeta-functions in the expected form of the result

$$
\Delta E_{6-\text { loop }}=c_{1}+c_{2} \zeta(3)+c_{3} \zeta(5)+c_{4} \zeta(7)+c_{5} \zeta(9)+c_{6} \zeta^{2}(3)+c_{7} \zeta(3) \zeta(5)
$$

and

$$
\begin{aligned}
\Delta E_{7-\text { loop }}= & c_{1}+c_{2} \zeta(3)+c_{3} \zeta(5)+c_{4} \zeta(7)+c_{5} \zeta(9)+c_{6} \zeta(11)+c_{7} \zeta^{2}(3)+ \\
& c_{8} \zeta(3) \zeta(5)+c_{9} \zeta(3) \zeta(7)+c_{10} \zeta^{3}(3)+c_{11} \zeta^{2}(5)
\end{aligned}
$$

assuming, as was the case for lower orders, that the coefficients are integers.

The plan of the paper is as follows. The anomalous dimension of the Konishi operator corresponds to the finite volume energy of a two particle scattering state. In section 2 we derive the ABA momentum and energy of this state up to seven loops. In section 3 we express the leading vacuum polarization effects in terms of the scattering matrix and evaluate their weak coupling expansion, thus obtaining the integrand of the multiparticle Lüscher correction formula. These expressions are subject to a consistency check in section 4 , where we also compute analytically the highest transcendental part of the answer which will be an important cross-check for our numerical approach. In section 5 we discuss the semi-numerical computation of our result. Finally we provide the full dimension of the Konishi operator up to seven loops in section 6 and make some concluding remarks.

\footnotetext{
${ }^{1}$ This is akin to the method used in [16]. In our case, however, a purely numerical summation does not suffice to attain the required accuracy and we use several orders of additional asymptotic analysis.
} 


\section{Asymptotic Bethe Ansatz up to 7-loops}

Finite volume energy corrections of multiparticle states have two sources: momentum quantization and vacuum polarization. Asymptotic BA determines momentum quantization and captures all finite size corrections, which are polynomial in the inverse of the volume. In contrast, vacuum polarization effects are exponentially supressed and their leading term is described by Lüscher-type formulas.

The Konishi operator corresponds to a two-particle state in volume $L=2$. These particles scatter diagonally on each other and the asymptotical BA equation quantizes their momenta as:

$$
e^{i p L}=S(p,-p) .
$$

Once the momenta are determined the asymptotic energy of the state can be calculated from the dispersion relation ${ }^{2}$

$$
\epsilon(p)=\sqrt{1+16 g^{2} \sin ^{2} \frac{p}{2}}
$$

as

$$
E_{A B A}(p)=\epsilon(p)+\epsilon(-p)=2 \epsilon(p) .
$$

The scattering matrix takes the form:

$$
S(p,-p)=\frac{\epsilon(p) \cot \frac{p}{2}+i}{\epsilon(p) \cot \frac{p}{2}-i} e^{-2 i p} e^{2 i \theta(p,-p)},
$$

where the dressing phase, $\theta(p,-p)$, is parameterized in the following manner:

$$
\theta\left(x_{1}, x_{2}\right)=\chi\left(x_{1}^{+}, x_{2}^{+}\right)+\chi\left(x_{1}^{-}, x_{2}^{-}\right)-\chi\left(x_{1}^{+}, x_{2}^{-}\right)-\chi\left(x_{1}^{-}, x_{2}^{+}\right)
$$

with

$$
x^{ \pm}(p)=\frac{1}{4 g}\left(\cot \frac{p}{2} \pm i\right)(1+\epsilon(p)) .
$$

The functions $\chi$ have a double expansion

$$
\chi\left(x_{1}, x_{2}\right)=-\sum_{r=2}^{\infty} \sum_{s>r} \frac{c_{r, s}(g)}{(r-1)(s-1)}\left[\frac{1}{x_{1}^{r-1} x_{2}^{s-1}}-\frac{1}{x_{1}^{s-1} x_{2}^{r-1}}\right]
$$

and the $c_{r, s}$ coefficients,

$$
c_{r, s}(g)=(r-1)(s-1) 2 \cos \left(\frac{\pi}{2}(s-r-1)\right) \int_{0}^{\infty} d t \frac{J_{r-1}(2 g t) J_{s-1}(2 g t)}{t\left(e^{t}-1\right)},
$$

are written such that in the weak coupling limit $(g \rightarrow 0)$ the integrals can be easily evaluated to yield:

$$
\begin{aligned}
& c_{2,3}(g)=4 \zeta_{3} g^{3}-40 \zeta_{5} g^{5}+420 \zeta_{7} g^{7}-4704 \zeta_{9} g^{9}+\ldots \\
& c_{2,5}(g)=-8 \zeta_{5} g^{5}+168 \zeta_{7} g^{7}+\ldots \\
& c_{3,4}(g)=24 \zeta_{5} g^{5}-420 \zeta_{7} g^{7}+\ldots
\end{aligned}
$$

\footnotetext{
${ }^{2}$ The 't Hooft coupling is reletad to $g$ as $g=\frac{\sqrt{\lambda}}{4 \pi}$.
} 
We solve the asymptotic BA equation (2.1) iteratively:

$$
\begin{aligned}
p= & +\frac{2 \pi}{3}-\sqrt{3} g^{2}+\frac{9}{2} \sqrt{3} g^{4}-24 \sqrt{3}\left(1+\zeta_{3}\right) g^{6}+\frac{\sqrt{3}}{4}\left(671+960\left(\zeta_{3}+\zeta_{5}\right)\right) g^{8} \\
& -\frac{6 \sqrt{3}}{5}\left(1269+1880 \zeta_{3}+2060 \zeta_{5}+2100 \zeta_{7}\right) g^{10} \\
& +6 \sqrt{3}\left(2643+12 \zeta_{3}\left(311+8 \zeta_{5}\right)+4072 \zeta_{5}+4452 \zeta_{7}+4704 \zeta_{9}\right) g^{12} .
\end{aligned}
$$

By plugging back this asymptotic momentum into the dispersion relation we obtain the asymptotic energy of the Konishi state:

$$
\begin{aligned}
E_{B Y}=2 \epsilon(p)= & 2+12 g^{2}-48 g^{4}+336 g^{6}-12\left(235+24 \zeta_{3}\right) g^{8} \\
& +12\left(2209+360 \zeta_{3}+240 \zeta_{5}\right) g^{10} \\
& -12\left(22429+4608 \zeta_{3}+3672 \zeta_{5}+2520 \zeta_{7}\right) g^{12} \\
& +24\left(119885+24 \zeta_{3}\left(1211+6 \zeta_{3}\right)+24156 \zeta_{5}+19656 \zeta_{7}+14112 \zeta_{9}\right) g^{14}
\end{aligned}
$$

This provides the exact answer up to $g^{6}$ (three loops) as from four loops onwards wrapping corrections start to contribute.

\section{$3 \quad$ Lüscher type corrections}

The vacuum polarization effects show up in two different ways. First, particles polarize the vacuum in which they are moving, this is what we call the direct energy correction. Second, the polarized vacuum reacts back and modifies - via the asymptotic Bethe Ansatz - the particles momenta, this we call the BA modification. Let us analyze them one after one the other.

\subsection{Direct energy correction}

The polarized vacuum will contribute to the energy as

$$
\Delta E=-\sum_{Q=1}^{\infty} \int \frac{d q}{2 \pi} \operatorname{sir}\left(S_{Q 1}^{Q 1}(q, p) S_{Q 1}^{Q 1}(q,-p)\right) e^{-\tilde{\epsilon}_{Q}(q) L}+O\left(g^{16}\right) .
$$

Here we sum up for all bound-states of charge $Q$ of the mirror model, whose momenta are $q$. $S_{Q 1}^{Q 1}$ describes how these mirror bound-states scatter on the fundamental "Konishi" particle, while $\tilde{\epsilon}_{Q}(q)$ denotes their mirror energy. This expression is exact in the weak coupling limit up to the order $g^{14}$ since at the order $g^{16}$ double wrapping effects will contribute, too. In order to obtain the six- and seven-loop anomalous dimension of the Konishi operator we need to expand this expression up to $g^{14}$. Let us analyze the $g$ dependence of the various terms.

Exponential factor. The mirror energy has the following parametrization

$$
e^{-\tilde{\epsilon}_{Q}(q)}=\frac{z^{-}(q, Q)}{z^{+}(q, Q)} ; \quad z^{ \pm}(q, Q)=\frac{q+i Q}{4 g}\left(\sqrt{1+\frac{16 g^{2}}{q^{2}+Q^{2}}} \pm 1\right) .
$$


Matrix part of the scattering matrix. There are many representative of the Konishi operator and in the present paper we choose the $s u(2)$ one which we label by (1i). This choice has the advantage that only diagonal matrix elements will contribute to eq. (3.1). The scattering matrix can be factorized as

$$
S_{(\alpha \dot{\alpha})(1 \dot{1})}^{(\alpha \dot{\alpha})}\left(z^{ \pm}, x^{ \pm}\right)=S_{\text {scal }}\left(z^{ \pm}, x^{ \pm}\right) S_{\alpha 1}^{\alpha 1}\left(z^{ \pm}, x^{ \pm}\right) S_{\dot{\alpha} \dot{1}}^{\dot{\alpha} \dot{1}}\left(z^{ \pm}, x^{ \pm}\right)
$$

where $(\alpha, \dot{\alpha})$ labels the polarization of the mirror bound-states. For one single $s u(2 \mid 2)$ algebra the basis for boundstates can be represented in the superspace formalism as $\left(w_{3}^{j} w_{4}^{Q-j}, w_{3}^{j} w_{4}^{Q-2-j} \theta_{1} \theta_{2}, w_{3}^{j} w_{4}^{Q-1-j} \theta_{1}, w_{3}^{j} w_{4}^{Q-1-j} \theta_{2}\right)$, where $j$ takes values from 0 to $(Q, Q-2, Q-1, Q-1)$, respectively. See [5, 26] for the details. As the two $s u(2 \mid 2)$ factors are equivalent the contribution of the scattering matrix can be factored as

$$
\operatorname{sTr}\left(S_{Q 1}^{Q 1}(q, p) S_{Q 1}^{Q 1}(q,-p)\right)=S_{\text {scal }}(q, p) S_{\text {scal }}(q,-p) \operatorname{sTr}\left(S_{\text {mat }}^{s u(2)}(q, p) S_{\text {mat }}^{s u(2)}(q,-p)\right)^{2} .
$$

We use formulas from [5] and the following identities:

$$
S_{\alpha 1}^{\alpha 1}\left(z^{ \pm}, x^{ \pm}\right)=S_{1 \alpha}^{1 \alpha}\left(x^{\mp}, z^{\mp}\right)=S_{3 \bar{\alpha}}^{3 \bar{\alpha}}\left(x^{ \pm}, z^{ \pm}\right)
$$

where $\bar{\alpha}$ is the image of $\alpha$ under the transformation $1 \leftrightarrow 3,2 \leftrightarrow 4$, but it will not be important here as in the supertrace we sum over all states. From [5] we can extract the matrix elements, which turn out to be independent of $j$ :

$$
\begin{gathered}
w_{1}^{j} w_{2}^{Q-j} \rightarrow a_{5}^{5}(x, z) \equiv S 1\left(z, x^{ \pm}\right)=\frac{z^{+}-x^{+}}{z^{-}-x^{+}} \frac{\tilde{\eta}_{1}}{\eta_{1}} \\
w_{1}^{j} w_{2}^{Q-2-j} \theta_{3} \theta_{4} \rightarrow 2 a_{8}^{8}(x, z) \equiv S 2\left(z, x^{ \pm}\right)=\frac{z^{+}-x^{-}}{z^{-}-x^{+}} \frac{\left(1-x^{+} z^{-}\right)}{\left(1-x^{-} z^{-}\right)} \frac{x^{-}}{x^{+}} \frac{\tilde{\eta}_{1}}{\eta_{1}}\left(\frac{\tilde{\eta}_{2}}{\eta_{2}}\right)^{2} \\
w_{1}^{j} w_{2}^{Q-1-j} \theta_{3} \rightarrow a_{9}^{9}(x, z) \equiv S 3\left(z, x^{ \pm}\right)=\frac{z^{+}-x^{-}}{z^{-}-x^{+}} \frac{\tilde{\eta}_{1}}{\eta_{1}} \frac{\tilde{\eta}_{2}}{\eta_{2}} \\
w_{1}^{j} w_{2}^{Q-1-j} \theta_{4} \rightarrow \frac{1}{2}\left(a_{3}^{3}(x, z)+a_{9}^{9}(x, z)\right) \equiv S 4\left(z, x^{ \pm}\right)=\frac{z^{+}-x^{+}}{z^{-}-x^{+}} \frac{\left(1-x^{+} z^{-}\right)}{\left(1-x^{-} z^{-}\right)} \frac{x^{-}}{x^{+}} \frac{\tilde{\eta}_{1}}{\eta_{1}} \frac{\tilde{\eta}_{2}}{\eta_{2}} .
\end{gathered}
$$

The string frame factors appearing here can be written as $\frac{\tilde{\eta}_{1}}{\eta_{1}}=\sqrt{\frac{z^{-}}{z^{+}}}$and $\left(\frac{\tilde{\eta}_{2}}{\eta_{2}}\right)^{2}=\frac{x^{+}}{x^{-}}$. As in the Konishi state we have two particles with opposite momenta the factors $\frac{\tilde{\eta}_{2}}{\eta_{2}}$ will cancel:

$$
\begin{gathered}
\operatorname{sTr}\left(S_{\text {mat }}^{\text {su(2) }}\left(z, x^{ \pm}\right) S_{\mathrm{mat}}^{\text {su( }(2)}\left(z,-x^{\mp}\right)\right)=(Q+1) S 1\left(z, x^{ \pm}\right) S 1\left(z,-x^{\mp}\right) \\
+(Q-1) S 2\left(z, x^{ \pm}\right) S 2\left(z,-x^{\mp}\right)-Q S 3\left(z, x^{ \pm}\right) S 3\left(z,-x^{\mp}\right)-Q S 4\left(z, x^{ \pm}\right) S 4\left(z,-x^{\mp}\right) \\
=\frac{2\left(x^{-}-x^{+}\right)\left(z^{+}\left(-1-2 Q x^{+} z^{-}+z^{-} z^{+}\right)+x^{-} z^{-}\left(2 Q z^{+}+x^{+}\left(-1+z^{-} z^{+}\right)\right)\right) z^{-}}{\left(x^{-}+z^{-}\right)\left(z^{-}-x^{+}\right)\left(-1+x^{-} z^{-}\right)\left(1+x^{+} z^{-}\right) z^{+}} .
\end{gathered}
$$

Scalar factor. The scalar factor for the fundametal particles is

$$
S_{\mathrm{scal}}^{Q=1}(z, x)=\frac{z^{-}-x^{+}}{z^{+}-x^{-}} \frac{1-\frac{1}{z^{+} x^{-}}}{1-\frac{1}{z^{-} x^{+}}}
$$


and this is what we should fuse for the bound-state:

$$
S_{\mathrm{scal}}(z, x)=\prod_{i} S_{\mathrm{scal}}^{Q=1}\left(z_{i}, x\right)
$$

There is a nicer form, however, which does not depend on the individual constituents:

$$
S_{\text {scal }}^{-1}(z, x)=\Sigma_{Q, 1}^{2}(z, x) S^{s u(2)}(z, x)
$$

The $s u(2)$ scalar factor is defined as:

$$
S^{s u(2)}(z, x)=\frac{\left(z^{+}-x^{-}\right)\left(z^{+}-x^{+}\right)}{\left(z^{-}-x^{+}\right)\left(z^{-}-x^{-}\right)} \frac{\left(1-\frac{1}{z^{+} x^{-}}\right)\left(1-\frac{1}{z^{+} x^{+}}\right)}{\left(1-\frac{1}{z^{-} x^{+}}\right)\left(1-\frac{1}{z^{-} x^{-}}\right)}
$$

while for the case $\left|x^{ \pm}\right|>1$ following [27] we can write ${ }^{3}$

$$
\begin{aligned}
-i \log \Sigma_{Q, 1}(z, x)= & \Phi\left(z^{+}, x^{+}\right)-\Phi\left(z^{+}, x^{-}\right)-\Phi\left(z^{-}, x^{+}\right)+\Phi\left(z^{-}, x^{-}\right) \\
& -\frac{1}{2}\left[-\Psi\left(z^{+}, x^{+}\right)+\Psi\left(z^{+}, x^{-}\right)-\Psi\left(z^{-}, x^{+}\right)+\Psi\left(z^{-}, x^{-}\right)\right] \\
& +\frac{1}{2 i} \log \left[\frac{\left(z^{-}-x^{-}\right)\left(x^{+}-\frac{1}{z^{-}}\right)^{2}}{\left(z^{-}-x^{+}\right)\left(x^{+}-\frac{1}{z^{+}}\right)\left(x^{-}-\frac{1}{z^{+}}\right)}\right] .
\end{aligned}
$$

The functions $\Phi$ and $\Psi$ are defined in terms of the integrals

$$
\begin{aligned}
& \Phi\left(x_{1}, x_{2}\right)=i \oint_{C_{1}} \frac{d w_{1}}{2 \pi i} \oint_{C_{1}} \frac{d w_{2}}{2 \pi i} \frac{1}{w_{1}-x_{1}} \frac{1}{w_{2}-x_{2}} \log \frac{\Gamma\left(1+i g\left(w_{1}+w_{1}^{-1}-w_{2}-w_{2}^{-1}\right)\right)}{\Gamma\left(1-i g\left(w_{1}+w_{1}^{-1}-w_{2}-w_{2}^{-1}\right)\right)} \\
& \Psi\left(x_{1}, x_{2}\right)=i \oint_{C_{1}} \frac{d w_{2}}{2 \pi i} \frac{1}{w_{2}-x_{2}} \log \frac{\Gamma\left(1+i g\left(x_{1}+x_{1}^{-1}-w_{2}-w_{2}^{-1}\right)\right)}{\Gamma\left(1-i g\left(x_{1}+x_{1}^{-1}-w_{2}-w_{2}^{-1}\right)\right)} .
\end{aligned}
$$

We separate the rational part from the $\Psi$ and $\Phi$ functions as

$$
S_{\text {scal }}(z, x)=S_{0}(z, x) e^{i \sigma(z, x)}
$$

where

$$
S_{0}(z, x)=\frac{\left(z^{-}-x^{+}\right)^{2}\left(1-z^{-} x^{-}\right)}{\left(z^{+}-x^{-}\right)\left(z^{+}-x^{+}\right)\left(1-z^{-} x^{+}\right)}
$$

and

$$
\begin{aligned}
\sigma(z, x)= & 2\left(\Phi\left(z^{+}, x^{+}\right)-\Phi\left(z^{+}, x^{-}\right)-\Phi\left(z^{-}, x^{+}\right)+\Phi\left(z^{-}, x^{-}\right)\right) \\
& -\left(-\Psi\left(z^{+}, x^{+}\right)+\Psi\left(z^{+}, x^{-}\right)-\Psi\left(z^{-}, x^{+}\right)+\Psi\left(z^{-}, x^{-}\right)\right) .
\end{aligned}
$$

\footnotetext{
${ }^{3}$ We have to be careful as the conventions of [27] are different from ours. To turn into our conventions we made the replacements: $z^{ \pm} \rightarrow z^{\mp}$ and $x^{ \pm} \rightarrow x^{\mp}$.
} 
Weak coupling expansion. Our aim is to calculate the weak coupling expansion of $\Delta E$ for $L=2$. In so doing we decompose the integrand of the Lüscher correction (3.1)

$$
\Delta E=-\sum_{Q=1}^{\infty} \int \frac{d q}{2 \pi} P(q, Q) \Sigma(q, Q)
$$

into a simpler rational part

$$
P(q, Q)=\frac{4\left(x^{-}-x^{+}\right)^{2}\left(z^{+}\left(-1-2 Q x^{+} z^{-}+z^{-} z^{+}\right)+x^{-} z^{-}\left(2 Q z^{+}+x^{+}\left(-1+z^{-} z^{+}\right)\right)\right)^{2}}{\left(\left(z^{+}\right)^{2}-\left(x^{+}\right)^{2}\right)\left(\left(z^{+}\right)^{2}-\left(x^{-}\right)^{2}\right)\left(-1+\left(x^{-}\right)^{2}\left(z^{-}\right)^{2}\right)\left(1+\left(x^{+}\right)^{2}\left(z^{-}\right)^{2}\right)}\left(\frac{z^{-}}{z^{+}}\right)^{4}
$$

which contains both the matrix part and the rational part of the scalar factor, and into the more complicated $\Sigma$ part:

$$
\begin{aligned}
i \log \Sigma(q, Q)= & -2\left(\Phi\left(z^{+}, x^{+}\right)-\Phi\left(z^{+}, x^{-}\right)-\Phi\left(z^{-}, x^{+}\right)+\Phi\left(z^{-}, x^{-}\right)\right) \\
& -2\left(\Phi\left(z^{+},-x^{-}\right)-\Phi\left(z^{+},-x^{+}\right)-\Phi\left(z^{-},-x^{-}\right)+\Phi\left(z^{-},-x^{+}\right)\right) \\
& -\Psi\left(z^{+}, x^{+}\right)+\Psi\left(z^{+}, x^{-}\right)-\Psi\left(z^{-}, x^{+}\right)+\Psi\left(z^{-}, x^{-}\right) \\
& -\Psi\left(z^{+},-x^{-}\right)+\Psi\left(z^{+},-x^{+}\right)-\Psi\left(z^{-},-x^{-}\right)+\Psi\left(z^{-},-x^{+}\right) .
\end{aligned}
$$

We expand these functions in $g^{2}$ as

$$
\begin{aligned}
& P(q, Q)=P_{8}(q, Q) g^{8}+P_{10}(q, Q) g^{10}+P_{12}(q, Q) g^{12}+P_{14}(q, Q) g^{14}+\ldots \\
& \Sigma(q, Q)=1+\Sigma_{2}(q, Q) g^{2}+\Sigma_{4}(q, Q) g^{4}+\Sigma_{6}(q, Q) g^{6}+\ldots
\end{aligned}
$$

The expansion of the rational part is quite straightforward. In expanding the $\Psi$ and $\Phi$ functions we use the same method we used in [6]. The expansion of the $\Psi\left(x_{1}, x_{2}\right)$ functions for $\left|x_{2}\right|>1$ (string region) reads as follows

$$
\begin{aligned}
\Psi\left(x_{1}, x_{2}\right)= & -\frac{g}{x_{2}}\left(\Psi\left(1-i g\left(x_{1}+x_{1}^{-1}\right)\right)+\Psi\left(1+i g\left(x_{1}+x_{1}^{-1}\right)\right)\right) \\
& -\frac{i g^{2}}{2 x_{2}^{2}}\left(\Psi_{1}\left(1-i g\left(x_{1}+x_{1}^{-1}\right)\right)-\Psi_{1}\left(1+i g\left(x_{1}+x_{1}^{-1}\right)\right)\right) \\
& +g^{3}\left(\frac{1}{2 x_{2}}+\frac{1}{6 x_{2}^{3}}\right)\left(\Psi_{2}\left(1-i g\left(x_{1}+x_{1}^{-1}\right)\right)+\Psi_{2}\left(1+i g\left(x_{1}+x_{1}^{-1}\right)\right)\right) \\
& +\frac{i g^{4}}{6 x_{2}^{2}}\left(\Psi_{3}\left(1-i g\left(x_{1}+x_{1}^{-1}\right)\right)-\Psi_{3}\left(1+i g\left(x_{1}+x_{1}^{-1}\right)\right)\right) \\
& -\frac{g^{5}}{12 x_{2}}\left(\Psi_{4}\left(1-i g\left(x_{1}+x_{1}^{-1}\right)\right)+\Psi_{4}\left(1+i g\left(x_{1}+x_{1}^{-1}\right)\right)\right)+\ldots
\end{aligned}
$$

where $\Psi_{n}(x)=\left(\frac{d}{d x}\right)^{n+1}\left(\log (\Gamma(x))\right.$ are the standard polygamma functions. If $\left|x_{1}\right|>1$ then $\Phi\left(x_{1}, x_{2}\right)$ starts at $g^{6}$ and coincides with $\chi\left(x_{1}, x_{2}\right)$. In the opposite case using the identity $\Phi\left(x_{1}, x_{2}\right)=\Phi\left(0, x_{2}\right)-\Phi\left(x_{1}^{-1}, x_{2}\right)$, being valid if $\left|x_{1}\right| \neq 1$, we can calculate the leading expansion of $\Phi$ as

$$
\begin{aligned}
\Phi(0, x) & =\frac{2 \gamma_{E} g}{x}-2 \zeta_{3} g^{3}\left(\frac{3}{x}+\frac{1}{3 x^{3}}\right)+24 \zeta_{5} g^{5}\left(\frac{5}{3 x}+\frac{1}{3 x^{3}}\right)+\ldots \\
\Phi\left(x_{1}^{-1}, x_{2}\right) & =-2 \zeta_{3} g^{3}\left(\frac{x_{1}}{x_{2}^{2}}-\frac{x_{1}^{2}}{x_{2}}\right)+\ldots
\end{aligned}
$$


The $\Psi$ terms simplify, too:

$$
\begin{aligned}
& i \log \Sigma(q, Q)= \\
& 2 g\left(\frac{1}{x^{+}}-\frac{1}{x^{-}}\right)\left(4 \gamma_{E}+\Psi\left(1-i g u^{+}\right)+\Psi\left(1+i g u^{+}\right)+\Psi\left(1-i g u^{-}\right)+\Psi\left(1+i g u^{-}\right)\right) \\
& -g^{3}\left(\frac{1}{x^{+}}-\frac{1}{x^{-}}\right)\left(8 \zeta_{3} \cdot\left(3+\frac{1}{\left(z^{+}\right)^{2}}+\left(z^{-}\right)^{2}\right)\right. \\
& \left.+\Psi_{2}\left(1-i g u^{+}\right)+\Psi_{2}\left(1+i g u^{+}\right)+\Psi_{2}\left(1-i g u^{-}\right)+\Psi_{2}\left(1+i g u^{-}\right)\right) \\
& -\frac{g^{5}}{6}\left(\frac{1}{x^{+}}-\frac{1}{x^{-}}\right)\left(960 \zeta_{5}+\Psi_{4}\left(1-i g u^{+}\right)+\Psi_{4}\left(1+i g u^{+}\right)+\Psi_{4}\left(1-i g u^{-}\right)+\Psi_{4}\left(1+i g u^{-}\right)\right)
\end{aligned}
$$

where

$$
u^{ \pm}=z^{ \pm}+\frac{1}{z^{ \pm}}
$$

We have to plug back the parametrization of $x^{ \pm}$and $z^{ \pm}$and expand in $g$ to obtain $\Sigma_{2}$, $\Sigma_{4}$ and $\Sigma_{6}$. This leads to an explicit, however very complicated expression for the direct energy integrand in the form

$$
P_{12}(q, Q)+P_{10}(q, Q) \Sigma_{2}(q, Q)+P_{8}(q, Q) \Sigma_{4}(q, Q)
$$

for the 6-loop case and

$$
P_{14}(q, Q)+P_{12}(q, Q) \Sigma_{2}(q, Q)+P_{10}(q, Q) \Sigma_{4}(q, Q)+P_{8}(q, Q) \Sigma_{6}(q, Q)
$$

for 7-loop.

\subsection{Correction to the asymptotical BA}

The back reaction of the polarized vacuum is a change in the asymptotic BA equation:

$$
p L+i \log S(p,-p)-2 \pi=\Phi
$$

where

$$
\Phi=-\sum_{Q=1}^{\infty} \int \frac{d q}{2 \pi} \operatorname{sir}\left(\left(\partial_{q} S_{Q 1}^{Q 1}(q, p)\right) S_{Q 1}^{Q 1}(q,-p)\right) e^{-\tilde{\epsilon}_{Q}(q) L} .
$$

We can use eq. (3.33) to express $\delta p$ as

$$
\delta p=\frac{\Phi}{L+i \partial_{p} \log S(p,-p)}
$$

and with this correction the change in the energy is

$$
\Delta_{p} E=2 \partial_{p} E\left(p_{A B A}\right) \delta p
$$

As the derivative of the dispersion relaton is of order $g^{2}$ the momentum shift shows up one order higher in the energy formula. Thus it is sufficient to expand $\Phi$ up to the order $g^{12}$. 
We evaluated the differentiation in the integrand as follows:

$$
\begin{aligned}
& \mathrm{s} \operatorname{Tr}\left(\left(\partial_{q} S_{Q 1}^{Q 1}(q, p)\right) S_{Q 1}^{Q 1}(q,-p)\right)= \\
& \left(\partial_{q} S_{\text {scal }}(q, p)\right) S_{\text {scal }}(q,-p) \mathrm{s} \operatorname{Tr}\left(S_{\text {mat }}^{s u(2)}(q, p) S_{\text {mat }}^{s u(2)}(q,-p)\right)^{2} \\
& +2 S_{\text {scal }}(q, p) S_{\text {scal }}(q,-p) \mathrm{s} \operatorname{Tr}\left(\left(\partial_{q} S_{\text {mat }}^{s u(2)}(q, p)\right) S_{\text {mat }}^{s u(2)}(q,-p)\right) \mathrm{s} \operatorname{Tr}\left(S_{\text {mat }}^{s u(2)}(q, p) S_{\text {mat }}^{s u(2)}(q,-p)\right)
\end{aligned}
$$

where

$$
\begin{array}{r}
\operatorname{sTr}\left(\left(\partial_{q} S_{\text {mat }}^{s u(2)}\left(z, x^{ \pm}\right)\right) S_{\text {mat }}^{\text {su(2) }}\left(z,-x^{\mp}\right)\right)= \\
(Q+1)\left(\partial_{q} S 1\left(z, x^{ \pm}\right)\right) S 1\left(z,-x^{\mp}\right)+(Q-1)\left(\partial_{q} S 2\left(z, x^{ \pm}\right)\right) S 2\left(z,-x^{\mp}\right) \\
-Q\left(\partial_{q} S 3\left(z, x^{ \pm}\right)\right) S 3\left(z,-x^{\mp}\right)-Q\left(\partial_{q} S 4\left(z, x^{ \pm}\right)\right) S 4\left(z,-x^{\mp}\right) .
\end{array}
$$

In differentiating the scalar factor we need to differentiate the $S_{0}$ part and the $\sigma$ part separately:

$$
\partial_{q} S_{\text {scal }}(q, p)=\left(\partial_{q} S_{0}\left(z, x^{ \pm}\right)\right) e^{i \sigma\left(z, x^{ \pm}\right)}+S_{0}\left(z, x^{ \pm}\right) e^{i \sigma\left(z, x^{ \pm}\right)}\left(i \partial_{q} \sigma\left(z, x^{ \pm}\right)\right)
$$

Weak coupling expansion. We expand the modification of the BA as

$$
\Phi=g^{8} \Phi_{8}+g^{10} \Phi_{10}+g^{12} \Phi_{12}+\ldots
$$

which we write into the form

$$
\Phi=-\sum_{Q=1}^{\infty} \int \frac{d q}{2 \pi}(d P(q, Q) \Sigma(q, Q)+P(q, Q) d \Sigma(q, Q)) .
$$

Besides notations used before we introduced

$$
\begin{aligned}
d P(q, Q)= & \left(\partial_{q} S_{0}(q, p)\right) S_{0}(q,-p) \mathrm{s} \operatorname{Tr}\left(S_{\mathrm{mat}}^{s u(2)}\left(z, x^{ \pm}\right) S_{\mathrm{mat}}^{s u(2)}\left(z,-x^{\mp}\right)\right)^{2}+ \\
& \left.2 S_{0}(q, p)\right) S_{0}(q,-p) \mathrm{s} \operatorname{Tr}\left(\left(\partial_{q} S_{\mathrm{mat}}^{s u(2)}\left(z, x^{ \pm}\right)\right) S_{\mathrm{mat}}^{s u(2)}\left(z,-x^{\mp}\right)\right) \times \\
& \operatorname{sTr}\left(S_{\mathrm{mat}}^{s u(2)}\left(z, x^{ \pm}\right) S_{\mathrm{mat}}^{s u(2)}\left(z,-x^{\mp}\right)\right)
\end{aligned}
$$

and

$$
d \Sigma(q, Q)=i\left(\partial_{q} \sigma\left(z, x^{ \pm}\right)\right) \Sigma(q, Q) .
$$

We need to expand each function to third nontrivial order

$$
d P(q, Q)=d P(q, Q)_{8} g^{8}+d P(q, Q)_{10} g^{10}+d P(q, Q)_{12} g^{12} .
$$

In the $\sigma$ case we need to keep only the first non-trivial term which survives after symmetrization:

$\partial_{q} \sigma\left(z, x^{ \pm}\right)=\frac{g^{2}}{2}\left(\frac{1}{\left(x^{+}\right)^{2}}-\frac{1}{\left(x^{-}\right)^{2}}\right) \partial_{q}\left(\Psi_{1}\left(1-i g u^{+}\right)-\Psi_{1}\left(1+i g u^{+}\right)+\Psi_{1}\left(1-i g u^{-}\right)-\Psi_{1}\left(1+i g u^{-}\right)\right)$. 


\section{Cross-checks for the integrand}

In the previous sections we have derived the integrand of the six and seven loop contribution to the wrapping correction. This integrand consists of two quite distinct parts - the direct contribution to the energy which is essentially just the transfer matrix $E_{\text {direct }}(q, Q)$ and the wrapping contribution to the BY quantization condition $E_{\bmod }(q, Q)$. This term cannot be expressed in a simple form directly in terms of the transfer matrix and we had to evaluate the derivatives of S-matrix elements in order to calculate it (note that in contrast to the relativistic case we cannot exchange the derivative w.r.t. the first argument with minus the derivative w.r.t. the second argument). We also symmetrized both expressions w.r.t. $q$.

The obtained expression has thus the form

$$
\Delta E_{F \text {-term }}=-\frac{1}{2 \pi} \sum_{Q=1}^{\infty} \int_{-\infty}^{\infty} d q\left(E_{\text {direct }}(q, Q)-E_{\text {mod }}(q, Q)\right) .
$$

The above expression is, strictly speaking, just the $F$-term Lüscher correction. $\mu$-term corrections could in principle arise from the residues of the integrand at the dynamical poles ( $s$ - or $t$-channel physical poles). However at weak coupling one can argue (see [5]) that $\mu$-terms should not appear and hence the residues at the dynamical poles should cancel out after summation over $Q$. This indeed happened in our earlier computations both at four and at five loops. This constraint is a nontrivial cross check of the computation of both parts of the integrand, since the sum of the residues turns out to vanish only after we have included both the direct energy contribution $E_{\text {direct }}(q, Q)$ and the BY modification contribution $E_{\bmod }(q, Q)$.

Explicitly we find numerically that

$$
\sum_{Q=1}^{\infty} \sum_{q_{*} \in\left\{q_{5}, q_{6}, q_{7}, q_{8}\right\}} \operatorname{res}_{q=q_{*}} E_{\text {direct }}(q, Q)=\sum_{Q=1}^{\infty} \sum_{q_{*} \in\left\{q_{5}, q_{6}, q_{7}, q_{8}\right\}} \operatorname{res}_{q=q_{*}} E_{\bmod }(q, Q)
$$

where the dynamical poles in the upper half plane are

$$
q_{5,6}=\frac{\mp 1}{\sqrt{3}}+i(Q-1) \quad q_{7,8}=\frac{\mp 1}{\sqrt{3}}+i(Q+1) .
$$

Before we evaluate the full sum and integral numerically in the following section, it is also important to derive an independent analytical cross-check for our procedure of numerically fitting the coefficients of $\zeta$-functions for the final expression.

Experience with earlier four- and five-loop computations show that one can very easily derive analytically the coefficient of the single $\zeta$ function of maximal transcendentality. It comes just from evaluating the integral by residue on the 'kinematical pole' $q=i Q$ ignoring any other poles coming from the dressing phase. Moreover, this contribution arises only from the rational part of the direct energy contribution $E_{\text {direct }}(q, Q)$.

Explicitly, we compute $-i \operatorname{res}_{q=i Q} P_{12}(q, Q)$, perform a partial fraction expansion and look at the term proportional to $1 / Q^{9}$. In the present case we obtain

$$
-i \operatorname{res}_{q=i Q} P_{12}(q, Q)=-\frac{489888}{Q^{9}}+\frac{205632}{Q^{7}}+\ldots
$$


We expect that the coefficient of the maximally transcendental $\zeta(9)$ is -489888 . We will not assume it in the subsequent computation but will check at the end whether the numerically obtained coefficient of $\zeta(9)$ will agree with this predicition. Note, however, that the coefficients of $\zeta$ 's with lower transcendentality (like $\zeta(7)$ here) cannot be obtained in this way.

Analogous computations for the seven loop integrand give

$$
-i \operatorname{res}_{q=i Q} P_{14}(q, Q)=\frac{7318080}{Q^{11}}+\ldots
$$

thus predicting that the coefficient of $\zeta(11)$ in the final answer will be 7318080 . Again, we will not use this information in the numerical fits, but will check whether this coefficient arises independently from the numerics. For the seven loop integrand we have also checked numerically the cancellation of dynamical poles.

\section{The evaluation of the multiparticle Lüscher correction}

We will now proceed to evaluate the expression (4.1). We will first give details for the six loop computation and then briefly comment on the modifications at seven loops.

It is easy to see that a natural variable for the integrand is $s=q / Q$ and one can see that the integrand approaches at large $Q$ a single scaling function of $s$ (times an appropriate power of $Q$ ). Hence we introduce

$$
f_{6-\text { loop }}(s, Q)=Q\left(E_{\text {direct }}(s Q, Q)-E_{\bmod }(s Q, Q)\right)
$$

and evaluate

$$
-\sum_{Q=1}^{\infty} \int_{0}^{\infty} \frac{d s}{\pi} f_{6-l o o p}(s, Q) .
$$

We first numerically evaluate using Mathematica the integrals from $Q=1$ up to $Q_{\max }=$ 2000 using 80 digit precision (we set PrecisionGoal ${ }^{4}$ to 80 and WorkingPrecision to 100). By itself, this is a far too small $Q_{\max }$ to estimate the infinite sum with sufficient precision in order to reliably fit the coefficients of the $\zeta$ 's. We therefore supplement this numerical part of the computation by computing analytically the asymptotics of the integral around $Q=\infty$ to several orders and performing the sums on these asymptotics from $Q=Q_{\max }+1=2001$ to $Q=\infty$.

Explictly we find that the six-loop integral has the following asymptotic expansion at large $Q$ :

$$
f_{6-\text { loop }}(s, Q) \sim \sum_{n=0} \frac{1}{Q^{9+2 n}}\left(a_{n}(s)+b_{n}(s) \log \frac{1}{Q}+c_{n}(s) \log ^{2} \frac{1}{Q}\right) .
$$

We evaluated the first six orders (up to $1 / Q^{19}$ ) and integrated the coefficient functions $a_{n}(s), b_{n}(s), c_{n}(s)$ (two of these integrals could be done analytically by Mathematica but

\footnotetext{
${ }^{4}$ With hindsight, setting AccuracyGoal would be a more efficient option.
} 
80-digit numerical evaluation was faster and just as reliable). In this way we obtained the large $Q$ expansion of the final summands

$$
\sum_{n=0}^{5} \frac{1}{Q^{9+2 n}}\left(A_{n}+B_{n} \log \frac{1}{Q}+C_{n} \log ^{2} \frac{1}{Q}\right) .
$$

As a cross check, we verified that evaluating the above expression at our cut-off point $Q=2000$ agrees with the numerical integral for the same $Q$ up to $10^{-61}$. Now we sum the asymptotic expression (5.4) from $Q=2001$ to $\infty$ and add it to the numerical sum.

We use EZ-Face [28] to express the resulting number as a linear combination with integer coefficients. Using 44 digits precision we obtained

$$
\begin{aligned}
\Delta E_{6-\text { loop }}= & 261468-207360 \zeta(3)+156384 \zeta(5)+105840 \zeta(7)-489888 \zeta(9) \\
& +155520 \zeta(3) \zeta(5)-20736 \zeta^{2}(3) .
\end{aligned}
$$

We see that the the coefficient of $\zeta(9)$ obtained from the above semi-numerical procedure exactly agrees with the analytical prediction of previous section.

In fact our numerical expression for $\Delta E$ obtained using $Q_{\max }=2000$ and subsequent six orders of asymptotic expansion agrees with the above analytical expression up to $10^{-59}$ (this is more than 60 decimal digits agreement as $\Delta E=-42127.1014157 \ldots$.). In order to make a further test, we evaluated an additional seventh order of the asymptotic expansion. Incorporating its contribution to the numerical expression for $\Delta E$ increased its agreement with the analytical expression (5.5) up to $10^{-66}$. Finally, let us note that if we were to keep only the numerical sum up to $Q_{\max }=2000$ without the asymptotic expansions, the precision would be much too small to fit the $\zeta$ functions. Indeed the deviation of this sum from the exact answer starts already at order $10^{-20}$.

The evaluation of the seven loop wrapping correction proceeded along the same lines. We again evaluated the integrals numerically from $Q=1$ to $Q=2000$ using this time slightly different settings (we set now AccuracyGoal to 70 and WorkingPrecision to 90). We could not increase these settings due to some quirks in Mathematica 8. Indeed, at the end of the computation we found that Mathematica 8 did not evaluate the integrals for 24 values of $Q$, so we had to evaluate these remaining integrals using Mathematica 7.

Subsequently, we evaluated 9 orders of asymptotic expansion, the reason being that for the seven loop case we needed to fit 11 coefficients in contrast to 7 at the six loop level, and hence we needed maximal numerical accuracy. We thus evaluated

$$
\sum_{n=0}^{8} \frac{1}{Q^{9+2 n}}\left(A_{n}+B_{n} \log \frac{1}{Q}+C_{n} \log ^{2} \frac{1}{Q}+D_{n} \log ^{3} \frac{1}{Q}\right)
$$

in a similar manner as outlined above. The above expression evaluated for $Q=1999$ agreed with the numerical integral for that value of $Q$ up to $10^{-75}$. We then summed the above asymptotic expression from $Q=2001$ to $Q=\infty$ and added the result to the sum of the numerical integrals computed earlier.

In order to fit the coefficients of the $\zeta$ 's, it is advantageous to reduce the magnitude of the coefficients by factoring out an integer divisor. Experience with lower loop levels 
suggests to factor out 36. Now 65-digit precision in EZ-Face [28], allowed us to obtain the analytical answer:

$$
\begin{aligned}
\Delta E_{7-\text { loop }}=36 \cdot( & -139230+125904 \zeta(3)-22472 \zeta(5)-47964 \zeta(7)-32616 \zeta(9)+ \\
& +203280 \zeta(11)-11808 \zeta^{2}(3)+11424 \zeta(3) \zeta(5)-53760 \zeta(3) \zeta(7)+ \\
& \left.+3456 \zeta^{3}(3)-27600 \zeta^{2}(5)\right) .
\end{aligned}
$$

The deviation of the above expression from our semi-numerical answer appeared only at order $10^{-73}$, therefore right at the edge of our numerical precision. However, a key test of the above result is the coefficient of $\zeta(11)$ which was fitted to be $36 \cdot 203280=7318080$ and exactly coincides with our analytical prediction from the previous section.

\section{The final result and conclusions}

In order to compute the final anomalous dimension of the Konishi operator, it remains to add the six and seven loop wrapping corrections (5.5) and (5.7) to the relevant terms in the Asymptotic Bethe Ansatz answer (2.10). For completeness, we will present here the full result up to seven loops, incorporating also the four loop wrapping correction obtained in $[5]$

$$
\Delta E_{4-\text { loop }}=324+864 \zeta(3)-1440 \zeta(5)
$$

and the five loop one from [6]

$$
\Delta E_{5-\text { loop }}=-11340+2592 \zeta(3)-5184 \zeta^{2}(3)-11520 \zeta(5)+30240 \zeta(7)
$$

The final answer for the Konishi dimension is

$$
\begin{aligned}
\Delta= & 4+12 g^{2}-48 g^{4}+336 g^{6}+96(-26+6 \zeta(3)-15 \zeta(5)) g^{8} \\
& -96\left(-158-72 \zeta(3)+54 \zeta(3)^{2}+90 \zeta(5)-315 \zeta(7)\right) g^{10} \\
& -48\left(160+432 \zeta^{2}(3)-2340 \zeta(5)-72 \zeta(3)(-76+45 \zeta(5))-1575 \zeta(7)+10206 \zeta(9)\right) g^{12} \\
& +48\left(-44480-8784 \zeta^{2}(3)+2592 \zeta^{3}(3)-4776 \zeta(5)-20700 \zeta^{2}(5)\right. \\
& +24 \zeta(3)(4540+357 \zeta(5)-1680 \zeta(7))-26145 \zeta(7)-17406 \zeta(9)+152460 \zeta(11)) g^{14} .
\end{aligned}
$$

Our six loop result agrees with the computation of [25] obtained from FiNLIE thus being a very nontrivial cross check of the proposed finite set of nonlinear equations describing the spectrum of $\mathcal{N}=4 \mathrm{SYM}$. It would be very interesting to compare this result with a direct gauge theoretical computations along the lines of [22]. It would be also very interesting to analytically verify our seven loop result, as in this case the identification was performed at the edge of computational precision (however with a nontrivial consistency check of the analytically known coefficient of $\zeta(11)$ ). Finally let us note that extending the result for Konishi to eight loop order would be conceptually quite important, as at that order double wrapping effects will appear. These phenomena were analyzed for the nonprotected vacuum state in the $\gamma$-deformed theories in [29] but they are beyond the reach of the Lüscher correction formulas for excited states known up to now. 


\section{Acknowledgments}

We thank Gregory Korchemsky for suggesting this project to us. We are grateful to the authors of [25] for comunicating to us their result prior to publication, and to Dima Volin, Kolya Gromov and Sergey Frolov for discussions leading to the elimination of a discrepancy between our results. ZB thanks for the hospitality during the CQUeST-IEU Focus program on Finite-size Technology in Low Dimensional Quantum System (VI). ZB was supported by an MTA Lendület grant and by OTKA K81461.

Open Access. This article is distributed under the terms of the Creative Commons Attribution License which permits any use, distribution and reproduction in any medium, provided the original author(s) and source are credited.

\section{References}

[1] N. Beisert and M. Staudacher, Long-range PSU $(2,2 \mid 4)$ Bethe Ansatze for gauge theory and strings, Nucl. Phys. B 727 (2005) 1 [hep-th/0504190] [INSPIRE].

[2] J. Ambjørn, R.A. Janik and C. Kristjansen, Wrapping interactions and a new source of corrections to the spin-chain/string duality, Nucl. Phys. B 736 (2006) 288 [hep-th/0510171] [INSPIRE].

[3] F. Fiamberti, A. Santambrogio, C. Sieg and D. Zanon, Wrapping at four loops in $N=4$ SYM, Phys. Lett. B 666 (2008) 100 [arXiv:0712.3522] [inSPIRE].

[4] V. Velizhanin, The four-loop anomalous dimension of the Konishi operator in $N=4$ supersymmetric Yang-Mills theory, JETP Lett. 89 (2009) 6 [arXiv:0808.3832] [INSPIRE].

[5] Z. Bajnok and R.A. Janik, Four-loop perturbative Konishi from strings and finite size effects for multiparticle states, Nucl. Phys. B 807 (2009) 625 [arXiv:0807.0399] [INSPIRE].

[6] Z. Bajnok, A. Hegedus, R.A. Janik and T. Lukowski, Five loop Konishi from AdS/CFT, Nucl. Phys. B 827 (2010) 426 [arXiv:0906.4062] [INSPIRE].

[7] Z. Bajnok and O. el Deeb, 6-loop anomalous dimension of a single impurity operator from AdS/CFT and multiple zeta values, JHEP 01 (2011) 054 [arXiv:1010.5606] [INSPIRE].

[8] N. Gromov, V. Kazakov, A. Kozak and P. Vieira, Exact Spectrum of Anomalous Dimensions of Planar $N=4$ Supersymmetric Yang-Mills Theory: TBA and excited states, Lett. Math. Phys. 91 (2010) 265 [arXiv:0902.4458] [INSPIRE].

[9] D. Bombardelli, D. Fioravanti and R. Tateo, Thermodynamic Bethe Ansatz for planar AdS/CFT: A Proposal, J. Phys. A 42 (2009) 375401 [arXiv:0902.3930] [InSPIRE].

[10] G. Arutyunov and S. Frolov, Thermodynamic Bethe Ansatz for the AdS $S_{5} \times S^{5}$ Mirror Model, JHEP 05 (2009) 068 [arXiv:0903.0141] [InSPIRE].

[11] G. Arutyunov, S. Frolov and R. Suzuki, Five-loop Konishi from the Mirror TBA, JHEP 04 (2010) 069 [arXiv: 1002.1711] [INSPIRE].

[12] J. Balog and A. Hegedus, 5-loop Konishi from linearized TBA and the XXX magnet, JHEP 06 (2010) 080 [arXiv: 1002.4142] [INSPIRE]. 
[13] A. Kotikov and L. Lipatov, DGLAP and BFKL equations in the $N=4$ supersymmetric gauge theory, Nucl. Phys. B 661 (2003) 19 [Erratum ibid. B 685 (2004) 405-407] [hep-ph/0208220] [INSPIRE].

[14] A. Kotikov, L. Lipatov, A. Rej, M. Staudacher and V. Velizhanin, Dressing and wrapping, J. Stat. Mech. 0710 (2007) P10003 [arXiv:0704.3586] [INSPIRE].

[15] Z. Bajnok, R.A. Janik and T. Lukowski, Four loop twist two, BFKL, wrapping and strings, Nucl. Phys. B 816 (2009) 376 [arXiv:0811.4448] [INSPIRE].

[16] T. Lukowski, A. Rej and V. Velizhanin, Five-Loop Anomalous Dimension of Twist-Two Operators, Nucl. Phys. B 831 (2010) 105 [arXiv:0912.1624] [INSPIRE].

[17] N. Gromov, V. Kazakov and P. Vieira, Exact Spectrum of Planar $\mathcal{N}=4$ Supersymmetric Yang-Mills Theory: Konishi Dimension at Any Coupling, Phys. Rev. Lett. 104 (2010) 211601 [arXiv:0906.4240] [INSPIRE].

[18] S. Frolov, Konishi operator at intermediate coupling, J. Phys. A 44 (2011) 065401 [arXiv: 1006.5032] [INSPIRE].

[19] N. Gromov, D. Serban, I. Shenderovich and D. Volin, Quantum folded string and integrability: From finite size effects to Konishi dimension, JHEP 08 (2011) 046 [arXiv: 1102.1040] [INSPIRE].

[20] R. Roiban and A. Tseytlin, Semiclassical string computation of strong-coupling corrections to dimensions of operators in Konishi multiplet, Nucl. Phys. B 848 (2011) 251 [arXiv: 1102.1209] [INSPIRE].

[21] B.C. Vallilo and L. Mazzucato, The Konishi multiplet at strong coupling, JHEP 12 (2011) 029 [arXiv:1102.1219] [INSPIRE].

[22] B. Eden, P. Heslop, G.P. Korchemsky, V.A. Smirnov and E. Sokatchev, Five-loop Konishi in $N=4$ SYM, Nucl. Phys. B 862 (2012) 123 [arXiv:1202.5733] [INSPIRE].

[23] N. Gromov, V. Kazakov, S. Leurent and D. Volin, Solving the AdS/CFT Y-system, JHEP 07 (2012) 023 [arXiv:1110.0562] [INSPIRE].

[24] J. Balog and A. Hegedus, Hybrid-NLIE for the AdS/CFT spectral problem, JHEP 08 (2012) 022 [arXiv: 1202.3244] [INSPIRE].

[25] S. Leurent, D. Serban and D. Volin, Six-loop Konishi anomalous dimension from the $Y$-system arXiv:1209.0749 [INSPIRE].

[26] G. Arutyunov and S. Frolov, The S-matrix of String Bound States, Nucl. Phys. B 804 (2008) 90 [arXiv:0803.4323] [INSPIRE].

[27] G. Arutyunov and S. Frolov, The Dressing Factor and Crossing Equations, J. Phys. A 42 (2009) 425401 [arXiv:0904.4575] [InSPIRE].

[28] http://oldweb.cecm.sfu.ca/projects/EZFace/.

[29] C. Ahn, Z. Bajnok, D. Bombardelli and R.I. Nepomechie, TBA, NLO Lüscher correction and double wrapping in twisted AdS/CFT, JHEP 12 (2011) 059 [arXiv:1108.4914] [INSPIRE]. 